Proyecciones

Vol. 26, No 3, pp. 269-279, December 2007.

Universidad Católica del Norte

Antofagasta - Chile

\title{
A NOTE ON KKT-INVEXITY IN NONSMOOTH CONTINUOUS-TIME OPTIMIZATION
}

\author{
VALERIANO ANTUNES DE OLIVEIRA * \\ UNIVERSIDADE ESTADUAL PAULISTA, BRASIL \\ MARKO ANTONIO ROJAS-MEDAR ${ }^{\dagger}$ \\ UNIVERSIDAD DEL BÍO BÍO, CHILE \\ and \\ ADILSON J.V.BRANDÃO \\ UNIVERSIDADE FEDERAL DO ABC, BRASIL
}

Received : November 2006. Accepted : October 2007

\begin{abstract}
We introduce the notion of KKT-invexity for nonsmooth continuoustime nonlinear optimization problems and prove that this notion is a necessary and sufficient condition for every KKT solution to be a global optimal solution.
\end{abstract}

AMS Subject Classification: 90C26, 90C30, 90C46.

Key words and phrases : Nonsmooth continuous-time optimization, KKT conditions, KKT-invexity.

\footnotetext{
${ }^{*}$ Supported by CNPq-Brazil, Grant 141168/2003-0.

${ }^{\dagger}$ Partially supported by CGCI MECD-DGU Brazil/Spain, grant 2137-05-5, and by D.G.E.S. and M. C. y T. (Spain) MTM 2007-63432

${ }^{\ddagger}$ Partially supported by Instituto do Milenio-IM-AGIMB.
} 


\section{INTRODUCTION}

We regard the continuous-time nonlinear programming problem

$$
\left.\begin{array}{ll}
\operatorname{minimize} & \phi(x)=\int_{0}^{T} f(t, x(t)) d t, \\
\text { subject to } & g(t, x(t)) \leq 0 \text { a.e. in }[0, T], x \in X .
\end{array}\right\}
$$

Here $X$ is a nonempty open convex subset of the Banach space $L_{\infty}^{n}[0, T], \phi$ : $X \rightarrow \mathbf{R}, f(t, x(t))=\xi(x)(t), g(t, x(t))=\gamma(x)(t), \xi: X \rightarrow \Lambda_{1}^{1}[0, T]$ and $\gamma: X \rightarrow \Lambda_{1}^{m}[0, T]$, where $L_{\infty}^{n}[0, T]$ denotes the space of all $n$-dimensional vector-valued Lebesgue measurable functions defined on the compact interval $[0, T] \subset \mathbf{R}$, which are essentially bounded, with norm $\|\cdot\|_{\infty}$ defined by

$$
\|x\|_{\infty}=\max _{1 \leq j \leq n} \operatorname{ess} \sup \left\{\left|x_{j}(t)\right|, 0 \leq t \leq T\right\},
$$

where for each $t \in[0, T], x_{j}(t)$ is the $j$ th component of $x(t) \in \mathbf{R}^{n}$ and $\Lambda_{1}^{m}[0, T]$ denotes the space of all $m$-dimensional vector functions which are essentially bounded and Lebesgue measurable, defined on $[0, T]$, with the norm $\|\cdot\|_{1}$ defined by

$$
\|y\|_{1}=\max _{1 \leq j \leq m} \int_{0}^{T}\left|y_{j}(t)\right| d t
$$

The continuous problem was first investigated in 1953 by Bellman in [1]. He studied a type of optimization problem, which is now known as a continuous-time linear problem. After that, various authors have studied more general continuous-time problems, regarding, for example, nonlinear problems. In [9], Zalmai obtained Karush-Kuhn-Tucker conditions of optimality. The results by Zalmai are natural generalizations of the KKT conditions in finite dimension. The nonsmooth problem was considered, for instance, in Brandão et al. [2] and Rojas-Medar et al. [7]. A good list of references about continuous-time problems can be found in [9].

The notion of invexity was introduced in [4] by Hanson. This concept, which generalize convexity, is important on getting sufficient conditions of optimality. In the work [5], Martin relaxed invexity. He introduced the notion of KKT-invexity (in fact he called it KT-invexity), which is (like invexity) a sufficient condition for a KKT point to be a global minimizer. But what is interesting in the Martin's result is that KKT-invexity is also a necessary condition of optimality. Martin showed that every KKT point 
is a global minimizer if and only if the problem is KKT-invex. In [6], de Oliveira and Rojas-Medar obtained a similar result for the continuous-time problem, but with smooth functions. In this work we generalize the result of de Oliveira and Rojas-Medar for the nonsmooth case.

\section{ASSUMPTIONS AND NOTATION}

Let $V$ be an open convex subset of $\mathbf{R}^{n}$ containing the set $\left\{x(t) \in \mathbf{R}^{n}: x \in\right.$ $X, t \in[0, T]\}$.

We assume that $f$ and $g_{i}$ (the $i$ th component of $g$ ), $i \in I=\{1,2, \ldots, m\}$, are real functions defined on $V \times[0, T]$.

The functions $t \mapsto f(t, x(t))$ and $t \mapsto g(t, x(t))$ are assumed to be Lebesgue measurable and integrable for all $x \in X$.

We assume that, given $a \in V$, there exist an $\varepsilon>0$ and a positive number $k$ such that for all $t \in[0, T]$, and for all $x, y \in a+\varepsilon B$ (B denotes the unit ball of $\mathbf{R}^{n}$ ) we have $|f(t, x)-f(t, y)| \leq k\|x-y\|$. Similar hypotheses are assumed for $g_{i}, i \in I$. Hence, $f(t, \cdot)$ and $g_{i}(t, \cdot), i \in I$, are locally Lipschitz on $V$ throughout $[0, T]$.

Let $x \in X$ and $h \in L_{\infty}^{n}[0, T]$. We denote by $\phi^{\circ}(x ; h)$ and $g_{i}^{\circ}(t, x(t) ; h(t)), i \in$ $I$, the Clarke generalized directional derivative of $\phi$ and $g_{i}, i \in I$, at $x$ on the direction $h$, respectively. See Clarke [3] for more details.

Let $\mathbf{F}$ be the set of all feasible solutions of (CNP) (which we suppose nonempty), i.e.,

$$
\mathbf{F}=\{x \in X: g(t, x(t)) \leq 0 \text { a.e. in }[0, T]\} .
$$

Given $x \in \mathbf{F}$, we denote by $A_{i}(x)$ the subset of $[0, T]$ where the $i$ th constraint is active, i.e.,

$$
A_{i}(x)=\left\{t \in[0, T]: g_{i}(t, x(t))=0\right\} .
$$

\section{INVEX CHARACTERIZATION OF KKT SOLUTIONS}

In [5] Martin introduced the notion of KKT-invexity for mathematical programming problems and proved that every KKT point is a global minimizer if and only if the problem is KKT-invex. In this section we extend this concept for (CNP) and get a similar result.

Definition 3.1. We say that (CNP) is Karush-Kuhn-Tucker invex (or KKTinvex) if there exists a function $\eta:[0, T] \times V \times V \rightarrow \mathbf{R}^{n}$ such that 
$\eta(t, x(t), y(t)) \in L_{\infty}^{n}[0, T]$

$$
\phi(x)-\phi(y) \geq \phi^{\circ}(y ; \eta(x, y))
$$

and

$$
-g_{i}^{\circ}\left(t, y(t) ; \eta(t, x(t), y(t)) \geq 0 \text { a.e. in } A_{i}(y), i \in I,\right.
$$

for all $x, y \in \mathbf{F}$.

Remark 3.2. By $\eta(x, y)$ in (3.1) we mean the map from $X \times X$ into $L_{\infty}^{n}[0, T]$ given by $\eta(x, y)(t)=\eta(t, x(t), y(t))$.

Remark 3.3. The definition of invexity differs from the KKT-invexity one by the requirement that $g_{i}(t, x(t))-g_{i}(t, y(t)) \geq g_{i}^{\circ}(t, y(t) ; \eta(t, x(t), y(t))$ a. e. in $A_{i}(y), i \in I$, instead of (3.2).

Definition 3.4. We say that $y \in \mathbf{F}$ is a Karush-Kuhn-Tucker solution (or KKT solution) of (CNP) if there exist $\lambda_{i} \in L_{\infty}[0, T], i \in I$, such that

$$
\begin{aligned}
& \phi^{\circ}(y ; h)+\int_{0}^{T} \sum_{i \in I} \lambda_{i}(t) g_{i}^{\circ}(t, y(t) ; h(t)) d t \geq 0 \forall h \in L_{\infty}^{n}[0, T], \\
& \lambda_{i}(t) g_{i}(t, y(t))=0 \text { a.e. in }[0, T], i \in I, \\
& \lambda_{i}(t) \geq 0 \text { a.e. in }[0, T], i \in I .
\end{aligned}
$$

Definition 3.5. We say that $y \in \mathbf{F}$ is a global optimal solution of (CNP) if $\phi(x) \geq \phi(y)$ for all $x \in \mathbf{F}$.

In the next example we study a KKT-invex problem which is not an invex one, where hold the property that every KKT solution is a global optimal solution. So, this example shows that invexity, despite being sufficient, is not a necessary condition to hold such property.

Example 3.6. Let us consider the following nonlinear continuous-time problem:

$$
\begin{array}{ll}
\text { minimize } & \phi(x)=\int_{0}^{2} f(x(t)) d t \\
\text { subject to } & g(x(t)) \leq 0 \text { a.e. in }[0,2], x \in L_{\infty}[0,2],
\end{array}
$$


where $f, g: \mathbf{R} \rightarrow \mathbf{R}$ are given respectively by

$$
f(x)=\left\{\begin{array}{l}
1-\exp (-x) \text { if } x \geq 0 \\
-x^{2} \text { if } x<0
\end{array}\right.
$$

and $g(x)=-x$. Let $x, h \in \mathbf{R}$. Is is easy to see that $f$ is Clarke regular (see [3]) and

$$
f^{\circ}(x ; h)=\left\{\begin{array}{l}
\exp (-x) h \text { if } x>0, \\
-2 x h \text { if } x<0, \\
h \text { if } x=0 \text { and } h \geq 0, \\
0 \text { if } x=0 \text { and } h<0 .
\end{array}\right.
$$

Also, $g^{\circ}(x ; h)=-h$ for all $x, h \in \mathbf{R}$.

Let $\bar{x}(t)=0 \in L_{\infty}[0,2]$ and $\bar{\lambda}(t)=1 \in L_{\infty}[0,2]$. We have that

$\phi^{\circ}(\bar{x} ; h)+\int_{0}^{2} \bar{\lambda}(t) g^{\circ}(\bar{x}(t) ; h(t)) d t=\int_{0}^{2}\left[f^{\circ}(0 ; h(t))-h(t)\right] d t \geq 0 \forall h \in L_{\infty}[0,2]$.

So $\bar{x}=0$ is a KKT solution. Let us suppose that $y(t)>0$ a.e. in $P \subseteq[0,2]$ is another KKT solution, where $P$ has positive measure. Then there exists $\lambda \in L_{\infty}[0,2], \lambda(t) \geq 0$ a.e. in $[0,2]$, satisfying

$$
\begin{gathered}
\phi^{\circ}(y ; h)+\int_{0}^{2} \lambda(t) g^{\circ}(y(t) ; h(t)) d t \geq 0 \forall h \in L_{\infty}[0,2], \\
\lambda(t) g(y(t))=0 \text { a.e. in }[0,2] .
\end{gathered}
$$

Let $\hat{h}:[0,2] \rightarrow \mathbf{R}$ be defined by

$$
\hat{h}(t)=\left\{\begin{aligned}
-1 & \text { if } t \in P, \\
0 & \text { if } t \notin P .
\end{aligned}\right.
$$

It is clear that $\hat{h} \in L_{\infty}[0,2]$. From (3.7) we see that $\lambda(t)=0$ a.e. in $P$. Therefore from (3.6) it comes

$$
\begin{aligned}
0 & \leq \phi^{\circ}(y ; \hat{h})+\int_{0}^{2} \lambda(t) g^{\circ}(y(t) ; \hat{h}(t)) d t \\
& =\phi^{\circ}(y ; \hat{h})-\int_{0}^{2} \lambda(t) \hat{h}(t) d t=-\int_{P} \exp (-y(t)) d t,
\end{aligned}
$$

what is an absurd. Thus $\bar{x}=0$ is the only KKT solution of this problem.

It is clear that $\phi(x) \geq \phi(0)$ for all $x \in \mathbf{F}=\left\{x \in L_{\infty}[0,2]\right.$ : $x(t) \geq 0$ a.e. in $[0,2]\}$. Thus every KKT solution is a global optimal solution. 
This problem is not invex. Indeed, if we assume that it is invex we get a contradiction as follows. Suppose that the problem is invex. Then there exist $\eta:[0,2] \times V \times V \rightarrow \mathbf{R}$ such that $t \mapsto \eta(t, x(t), y(t)) \in L_{\infty}[0,2]$,

$$
\phi(x)-\phi(y) \geq \int_{0}^{2} f^{\circ}(y(t) ; \eta(t, x(t), y(t))) d t
$$

and

$$
-x(t)+y(t) \geq-\eta(t, x(t), y(t)) \text { a.e. in }[0,2]
$$

for all $x, y \in L_{\infty}[0,2]$. Using the last inequality, it is not difficult to verify that $f^{\circ}(y(t) ; x(t)-y(t)) \leq f^{\circ}(y(t) ; \eta(t, x(t), y(t)))$ a.e. in $[0,2]$ for all $x, y \in$ $L_{\infty}[0,2]$. Hence

$$
\begin{aligned}
\phi(x)- & \phi(y)-\int_{0}^{2} f^{\circ}(y(t) ; x(t)-y(t)) d t \\
& \geq \phi(x)-\phi(y)-\int_{0}^{2} f^{\circ}(y(t) ; \eta(t, x(t), y(t))) d t \geq 0
\end{aligned}
$$

for all $x, y \in L_{\infty}[0,2]$. For $x(t)=0$ and $y(t)=t$ in $[0,2]$ we have

$$
\phi(x)-\phi(y)-\int_{0}^{2} f^{\circ}(y(t) ; x(t)-y(t)) d t=-4 \exp (-2)<0,
$$

which contradicts (3.9).

Now we show that this problem is KKT-invex. Define $\eta: V \times V \rightarrow \mathbf{R}$ by

$$
\eta(x, y)=\left\{\begin{array}{l}
\exp (y)(f(x)-f(y)) \text { if } y>0 \\
(-2 y)^{-1}(f(x)-f(y)) \text { if } y<0 \\
f(x)-f(y) \text { if } y=0
\end{array}\right.
$$

Let $x, y \in \mathbf{F}$ and $t \in[0,2]$. We have that

$f^{\circ}(y(t) ; \eta(x(t), y(t)))=\left\{\begin{array}{l}\exp (-y(t)) \eta(x(t), y(t))=f(x(t))-f(y(t)) \text { if } y(t)>0, \\ -2 y(t) \eta(x(t), y(t))=f(x(t))-f(y(t)) \text { if } y(t)<0, \\ \eta(x(t), y(t))=f(x(t))-f(y(t)) \text { if } y(t)=0\end{array}\right.$

so that

$$
\phi(x)-\phi(y)-\int_{0}^{2} f^{\circ}(y(t) ; \eta(x(t), y(t))) d t=0
$$

and for $t \in A(y)=\{t \in[0,2]: y(t)=0\}$,

$$
-g^{\circ}(y(t) ; \eta(x(t), y(t)))=1-\exp (-x(t)) \geq 0 .
$$

Therefore this problem is KKT-invex. 
Different of the finite dimensional case, here we need of a constraint qualification.

Definition 3.7. We say that the constraint $g$ satisfies $(C Q)$ at $y \in \mathbf{F}$ if there do not exist $u_{i} \in L_{\infty}[0, T], u_{i} \geq 0, i \in I$, not all zero, such that

$$
\sum_{i \in I} \int_{A_{i}(y)} u_{i}(t) g_{i}^{\circ}(t, y(t) ; h(t)) d t \geq 0 \forall h \in L_{\infty}^{n}[0, T] .
$$

Lemma 3.8. Let $y \in \mathbf{F}$ and assume that $g$ satisfies (CQ) at $y$. If $y$ is not a KKT solution of $(C N P)$ then there exists $h \in L_{\infty}^{n}[0, T]$ such that

$$
\begin{aligned}
& \phi^{\circ}(y ; h)<0, \\
& g_{i}^{\circ}(t, y(t) ; h(t)) \leq 0 \text { a.e. in } A_{i}(y), i \in I .
\end{aligned}
$$

Proof. If the system in (3.9) and (3.10) does not have a solution, particularly, the system

$$
\begin{aligned}
& \phi^{\circ}(y ; h)<0, \\
& \chi_{i}(t) g_{i}^{\circ}(t, y(t) ; h(t)) \leq 0 \text { a.e. in }[0, T], i \in I,
\end{aligned}
$$

does not have a solution, where $\chi_{i}:[0, T] \rightarrow \mathbf{R}$ is defined for each $i \in I$ by

$$
\chi_{i}(t)= \begin{cases}1 & \text { if } t \in A_{i}(y) \\ 0 & \text { if } t \notin A_{i}(y)\end{cases}
$$

It follows from Corollary 3.1 on page 134 of [8], that there exist $u_{0} \in \mathbf{R}$ and $u_{i} \in L_{\infty}[0, T], i \in I$, with $u_{0} \geq 0$ and $u_{i}(t) \geq 0$ a.e. in $[0, T], i \in I$, not all zero, such that

$$
u_{0} \phi^{\circ}(y ; h)+\int_{0}^{T} \sum_{i \in I} u_{i}(t) \chi_{i}(t) g_{i}^{\circ}(t, y(t) ; h(t)) d t \geq 0 \forall h \in L_{\infty}^{n}[0, T] .
$$

If $u_{0}=0$ we have a contradiction with the constraint qualification. Therefore $u_{0}>0$. Then dividing the expression in (3.11) by $u_{0}$ and defining $\lambda_{i}=u_{i} \chi_{i} / u_{0}, i \in I$, we obtain

$$
\phi^{\circ}(y ; h)+\int_{0}^{T} \sum_{i \in I} \lambda_{i}(t) g_{i}^{\circ}(t, y(t) ; h(t)) d t \geq 0 \forall h \in L_{\infty}^{n}[0, T] .
$$

Thus $y$ is a KKT solution, what contradicts the hypothesis. Hence, there exists $h \in L_{\infty}^{n}[0, T]$ satisfying (3.9) and (3.10). 
Theorem 3.9. Assume that $g$ satisfies $(C Q)$ at each $y \in \mathbf{F}$. Then, every KKT solution of (CNP) is a global optimal solution if and only if (CNP) is KKT-invex.

Proof. Necessity. Suppose that every KKT solution of (CNP) is a global optimal solution. Let $x, y \in \mathbf{F}$.

If $\phi(x)<\phi(y)$, then $y$ is not a global optimal solution, and so, by hypothesis, $y$ is not a KKT solution of (CNP). It follows from Lemma 3.8 that there exists $h \in L_{\infty}^{n}[0, T]$ satisfying (3.9) and (3.10). Set

$$
\alpha=\phi^{\circ}(y ; h)
$$

and

$$
\eta(t, x(t), y(t))=\{\phi(x)-\phi(y)\} \alpha^{-1} h(t) .
$$

Because of (3.9) we know that

$$
\{\phi(x)-\phi(y)\} \alpha^{-1}>0 .
$$

Hence

$$
\phi^{\circ}(y ; \eta(x, y))=\phi^{\circ}\left(y ;\{\phi(x)-\phi(y)\} \alpha^{-1} h\right)=\{\phi(x)-\phi(y)\} \alpha^{-1} \phi^{\circ}(y ; h),
$$

and therefore

$$
\phi^{\circ}(y ; \eta(x, y))=\phi(x)-\phi(y) .
$$

From (3.10) and (3.12) we get

$$
\begin{aligned}
g_{i}^{\circ}(t, y(t) ; \eta(t, x(t), y(t))) & =\{\phi(x)-\phi(y)\} \alpha^{-1} g_{i}^{\circ}(t, y(t) ; h(t)) \\
& \leq 0 \text { a.e. in } A_{i}(y), i \in I .
\end{aligned}
$$

Hence

$$
-g_{i}^{\circ}(t, y(t) ; \eta(t, x(t), y(t))) \geq 0 \text { a.e. in } A_{i}(y), i \in I \text {. }
$$

By (3.13) and (3.14) we conclude that for $\phi(x)<\phi(y)$, (CNP) is KKTinvex.

If $\phi(x) \geq \phi(y)$, define $\eta(t, x(t), y(t))=0$ a.e. in $[0, T]$. We have that

$$
\phi(x)-\phi(y)-\phi^{\circ}(y ; \eta(x, y)) \geq 0
$$

and

$$
g_{i}^{\circ}(t, y(t) ; \eta(t, x(t), y(t)))=0 \text { a.e in } A_{i}(y), i \in I .
$$


So, from (3.15) and (3.16) we see that (CNP) is KKT-invex.

In the cases above we do not define $\eta$ for $x, y \notin \mathbf{F}$. But we can take $\eta(t, x(t), y(t))=0$ when $x$ or $y$ is not feasible.

Sufficiency. Suppose that (CNP) is KKT-invex. Let $y \in \mathbf{F}$ be a KKT solution of $(\mathrm{CNP})$. It follows from $(3.4)$ that $\lambda_{i}(t)=0$ a.e. in $[0, T] \backslash$ $A_{i}(y), i \in I$. Then by (3.1), (3.2) and (3.5) we have

$$
\phi(x)-\phi(y)-\phi^{\circ}(y ; \eta(x, y))-\int_{0}^{T} \sum_{i \in I} \lambda_{i}(t) g_{i}^{\circ}(t, y(t) ; \eta(t, x(t), y(t))) d t \geq 0
$$

for all $x \in \mathbf{F}$. So, by (3.3) we obtain $\phi(x) \geq \phi(y)$ for all $x \in \mathbf{F}$, that is, $y$ is a global optimal solution of (CNP).

Remark 3.10. We observe that the assumption that $g$ satisfies (CQ) in the last theorem is necessary just on proving the "only if" part.

Remark 3.11. If $f(t, \cdot)$ and $g(t, \cdot)$ are Clarke regular at $y$, then (3.3) in Definition 3.4 can be replaced by

$$
0 \in \partial L(y, \lambda)
$$

where

$$
L(x, \lambda)=\int_{0}^{T}\left[f(t, x(t))+\sum_{i \in I} \lambda_{i}(t) g_{i}(t, x(t))\right] d t .
$$

Theorem 3.9, of course, is still valid.

Remark 3.12. It is well known that a convex function is Clarke regular. In particular, when $f(t, \cdot)$ and $g(t, \cdot)$ are convex at $y \in X$ throughout $[0, T]$ they are regular at $y$ and we have

$$
\partial L(y, \lambda)=\int_{0}^{T}\left[\partial f(t, y(t))+\sum_{i \in I} \lambda_{i} \partial g_{i}(t, y(t))\right] d t .
$$

An interesting open problem is to know if the relation (3.17) is still true when $f(t, \cdot)$ and $g(t, \cdot)$ are invex at $y$ throughout $[0, T]$. When we have a finite sum instead of an integral, we verified that this is true. 


\section{References}

[1] R. Bellman, Bottleneck problems and dynamics programming, Proc. Nat. Acad. Sci. U. S. A., 39, pp. 947-951, (1953).

[2] A. J. V. Brandão, M. A. Rojas-Medar and G.N. Silva, Nonsmooth continuous-time optimization problems: necessary conditions, Comp. Math. with Appl., 41, pp. 1477-1486, (2001).

[3] F. H. Clarke, Optimization and nonsmooth analysis, Classics in Applied Mathematics 5, SIAM, (1990).

[4] M. A. Hanson, On sufficiency of Kuhn-Tucker conditions, J. Math. Anal. Appl., 30, pp. 545-550, (1981).

[5] D. H. Martin, The essence of invexity, J. Optim. Theory Appl., 47, pp. 65-76, (1985).

[6] V. A. de Oliveira and M.A. Rojas-Medar, Continuous-time optimization problems involving invex functions, J. Math. Anal. Appl., 327, pp. 1320-1334, (2007).

[7] M. A. Rojas-Medar, A.J.V. Brandão and G.N. Silva, Nonsmooth continuous-time optimization problems: sufficient conditions, J. Math. Anal.Appl., 227, pp. 305-318, (1998).

[8] G. J. Zalmai, A continuous-time generalization of Gordan's transposition theorem, J. Math. Anal. Appl., 110, pp. 130-140, (1985).

[9] G. J. Zalmai, The Fritz John and Kuhn-Tucker optimality conditions in continuous-time nonlinear programming, J. Math. Anal. Appl., 110, pp. 503-518, (1985).

Note: This work was conduced while Valeriano A. de Oliveira was doing your Ph.D. course at the Department of Applied Mathematics, Scientific Computation, Statistics and Mathematics Institute, State University of Campinas, Campinas-SP, Brasil. Marko Rojas-Medar was the adviser and Adilson J. V. Brandão a colaborator. 


\section{Valeriano Antunes de Oliveira}

Departamento de Ciências de Computação e Estatística

Instituto de Biociências, Letras e Ciências Exatas

Universidade Estadual Paulista

Campus de São José do Río Preto

Rua Cristovão Colombo, 2265, CEP 15054-000, Jardim Nazareth

São José do Río Preto - SP

Brasil

e-mail : vantunes@webmail.ibilce.unesp.br

\section{Marko Antonio Rojas-Medar}

Departamento de Ciencias Básicas

Facultad de Ciencias

Universidad del Bío-Bío

Campus Fernando May

Casilla 447

Chillán

Chile

e-mail: marko@ueubiobio.cl

and

\section{Adilson José Vieira Brandão}

Departamento de Matemática

Centro de Matemática, Computação e Cognição

Universidade Federal do ABC

Rua Santa Adélia, 166, CEP 09210-170

Santo André-SP

Brasil

e-mail : adilson.brandao@ufabc.edu.br 